\title{
Short Communication: The Potential Effects of Scrophularia Striata Boiss on COVID-19
}

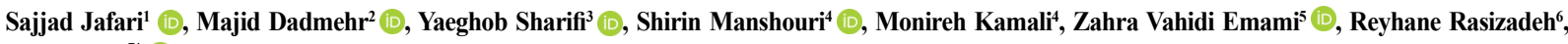
Farhad Seif ${ }^{7 *}$ (1)

1. Student Research Committee, Urmia University of Medical Sciences, Urmia, Iran.

2. Department of Traditional Medicine, School of Persian Medicine, Iran University of Medical Sciences, Tehran, Iran

3. Department of Microbiology and Virology, School of Medicine, Urmia University of Mdicinal Sciences, Urmia, Iran.

4. Rajaei Cardiovascular Medical and Research Center, Iran University of Medical Sciences, Tehran, Iran.

5. Department of Human Genetics, Qom Branch, Islamic Azad University, Qom, Iran.

6. Department of Virology, School of Medicine, Tabriz University of Medical Sciences, Tabriz, Iran.

7. Department of Immunology and Allergy, Iranian Academic Center for Education, Culture and Research, Tehran, Iran.

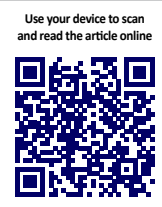

Citation Jafari S, Dadmehr M, Sharifi Y, Manshouri Sh, Kamali M, Vahidi Emami Z, et al. The Potential Effects of Scrophularia Striata Boiss on COVID-19. Immunoregulation. 2022; 4(2):69-72. http://dx.doi.org/10.32598/Immunoregulation.4.2.3

doi http://dx.doi.org/10.32598/Immunoregulation.4.2.3

\section{(i) (5)}

Article info:

Received: 21 Jul 2021

Accepted: 25 Aug 2021

Available Online: 01 Jan 2022

Keywords:

COVID-19, SARS-CoV-2, Scrophularia Striata Boiss, Cytokine, Quercetin

\begin{abstract}
A B S T RA C T
Severe Acute Respiratory Syndrome Coronavirus-2 (SARS-CoV-2) rapidly spread worldwide. The exact mechanisms involved in the pathogenesis of Coronavirus Disease 2019 (COVID-19) are not fully understood. In addition to classical treatment, herbal medicine may effectively manage COVID-19 symptoms and supportive therapy. Scrophularia striata Boiss is a perennial herbaceous plant with immunomodulatory and anti-inflammatory properties. The extract of $S$. striata Boiss has revealed immunomodulatory effects on reducing T helper 2 (Th2) cytokines, including IL-4 and IL-5, that may be used to manage allergic diseases and asthma. Scrophularia striata Boiss reduces IL-4, and IL-5 may alleviate allergic diseases and asthma. The ethyl acetate extract of $S$. striata Boiss inhibits the production of IL-1 $\beta$, TNF- $\alpha$, and PGE2. Quercetin inhibits proinflammatory mediators while increasing anti-inflammatory mediators. Ethanolic extract of $S$ striata can significantly reduce NO production in the isolated mouse peritoneal macrophages. Moreover, S. striata Boiss has 3 essential flavonoids: quercetin, isorhamnetin-3-O-rutinoside, and nepitrin. Of them, quercetin inhibits TNF- $\alpha$, IL-1 $\beta$, IL-6, PGE2, COX-2, and NO, whereas increases anti-inflammatory cytokines. Therefore, $S$. striata Boiss is recommended for clinical trial studies in patients with COVID-19 and other viral diseases.
\end{abstract}

\section{* Corresponding Author:}

Farhad Seif, PhD.

Address: Department of Immunology and Allergy, Iranian Academic Center for Education, Culture and Research, Tehran, Iran.

E-mail: farhad.seif@outlook.com 


\section{Introduction}

evere Acute Respiratory Syndrome Coronavirus 2 (SARS-CoV-2) originated in China in late 2019; it has rapidly spread worldwide and has become a global health emergency. During the Coronavirus Disease 2019 (COVID-19) pandemic, several antiviral drugs were introduced; however, they have not been approved as specific antiviral therapy for this disease so far. Accordingly, the mainstay of treatment in COVID-19 patients is the management of their symptoms and supportive treatment. Moreover, several innovative vaccines are being evaluated worldwide [1]. The exact mechanisms involved in the pathogenesis of COVID-19 are not fully understood; however, accumulating evidence suggests that hyperactivation of the immune system and cytokine storm may play a significant role in the development and course of the disease. The immune system is activated following the virus replication in the respiratory tract's epithelial cells. Elevated levels of proinflammatory cytokines at the site of tissue injury considerably correlate with bronchoalveolar damage and can lead to Acute Respiratory Distress Syndrome (ARDS). Recent studies reported the elevation of serum levels of inflammatory cytokines, including Interleukin (IL)-1 $\beta$, IL-6, IL-7, IL-8, IL-18, Interferon (IFN)- $\gamma$, Tumor Necrosis Factor (TNF)- $\alpha$ in COVID-19 patients [2,3].

Massive production and release of pro-inflammatory cytokines have been associated with tissue injuries in the heart, liver, kidneys, and other body organs and can eventually lead to shock, respiratory, and multiple organ failure. The overproduction of pro-inflammatory cytokines, such as IL1 , IL-6, IL-12, IFN- $\gamma$, and TNF- $\alpha$, affect the prognosis and outcome of most severe cases with COVID-19 [3]. Several therapeutic approaches, such as anti-cytokine therapy using monoclonal antibodies and signaling inhibitors, such as JAK-STAT inhibitors, have been suggested; however, they succeeded in a small number of critically ill patients [2]. In the COVID-19 epidemic, herbal-derived natural products' immunosuppressive and anti-inflammatory properties have been considered to reduce pro-inflammatory cytokines and modulate the immune system [1]. Interleukin-1 is among the most critical pro-inflammatory cytokines, mainly mediated in local and systemic inflammation in COVID-19. SARS-CoV-2 induces IL-1 in macrophages and mast cells, which in turn stimulates the secretion of other pro-inflammatory cytokines, such as IL- 6 and TNF- $\alpha$.

The release of IL-1 participates in both gastrointestinal and brain disorders and leads to hypotension and septic shock syndrome. Moreover, IL-1 increases Nitric Oxide (NO), releasing inflammatory products of arachidonic acid such as prostaglandins and thromboxane $\mathrm{A} 2$, which may participate in thromboembolic consequences $\mathrm{COV}$ ID-19 [4]. Furthermore, elevated circulating IL-6 predict adverse clinical outcomes in COVID-19 and considerably correlate with Bronchoalveolar inflammation and the need for mechanical ventilation. The effectiveness of IL-6 inhibitors, such as Tocilizumab, may implicate the critical role of IL-6 [2]. Moreover, IL-6 plays a crucial role in the pathogenesis of diabetes mellitus and cardiovascular diseases, i.e., the main comorbidities of COVID-19 [1].

Additionally, elevated serum levels of TNF- $\alpha$ were associated with pro-inflammatory responses mediated by IL-1 $\beta$ and IL-6. This cytokine is related to a poor prognosis and the disease severity; therefore, using TNF- $\alpha$ inhibitors, such as Etanercept or infliximab, may alleviate this life-threatening condition [2, 4]. In addition to classical treatment, herbal medicine may effectively manage COVID-19 symptoms and supportive therapy.

Scrophularia striata Boiss is a perennial herbaceous plant that belongs to the Scrophulariaceae family. It is frequently grown in western parts of Iran (locally known as Teshneh Dari). S. striata Boiss has immunomodulatory and anti-inflammatory activities. It has presented inhibitory effects on some pro-inflammatory mediators from macrophages. Different parts of this plant have been used since ancient times as traditional and folk remedies to treat conjunctivitis, otitis, scrophulas, scabies, eczema, psoriasis, gastritis, common cold, hemorrhoids, infectious wounds, and burns $[5,6]$. Interestingly, during the COVID-19 pandemic, using a decoction (hot water extract) of $S$. striata Boiss, one of the local plants, was frequent among people living in the western parts of Iran. Previous studies demonstrate that $S$. striata have antioxidant, anti-inflammatory, analgesic, antibacterial, antifungal, anticancer, antidepressant, neuroprotective, and wound healing properties [5]. The modulatory and anti-inflammatory effects of this plant have been studied frequently. Ethyl acetate extract of $S$. striata Boiss has demonstrated significant inhibitory effects on pro-inflammatory mediators such as IL- $1 \beta$, TNF- $\alpha$, and Prostaglandin E2 (PGE2) secretion in mice peritoneal macrophages induced by Lipopolysaccharide (LPS). This anti-inflammatory effect may be attributed to active ingredients, e.g. flavonoids in the plant extract [6]. Moreover, ethanolic extract of $S$. striata can significantly reduce NO production in the isolated mouse peritoneal macrophages and has an anti-inflammatory effect. Inhibiting NO can be a therapeutic strategy in inflammatory disorders, such as 
septic shock, which is associated with NO's overproduction [7]. Furthermore, the extract of S. striata Boiss has shown immunomodulatory effects on reducing T-helper 2 (Th2) cytokines, including IL-4 and IL-5, that may be used to manage allergic diseases and asthma [8].

Phytochemical investigations have shown the presence of different bioactive compounds, including cinnamic acid, three flavonoids (quercetin, isorhamnetin3-O-rutinoside, and nepitrin), \& one phenylpropanoid glycoside (acteoside 1) in this plant [6,9]. For example, quercetin is a flavonoid found abundantly in vegetables and fruits, i.e., anti-inflammatory, immunomodulatory, antioxidant, analgesic, and potential antiviral properties. Quercetin notably reduces inflammatory mediators production, including TNF- $\alpha$, IL-1 $\beta$, IL-6, PGE2, COX-2, and NO [10]. The mechanism of action Quercetin initially inhibits the production of inflammatory enzymes such as phospholipase A, Lipoxygenase (LOX), and Cyclooxygenase (COX). It limits induced inflammation via inhibition of Src- and Syk-mediated phosphatidylinositol-3-Kinase (PI3K)-(p85) tyrosine phosphorylation and subsequent Toll-Like Receptor 4 (TLR4)/MyD88/PI3K complex formation that limits activation of downstream signaling pathways in RAW 264.7 cells [11]. Quercetin significantly reduces the expression and activation of the Nuclear Factor kB (NF-kB) pathway; thus, preventing the accumulation of immune cells and suppressing proinflammatory cytokines including IL-1, TNF, IL-6. In contrast, it increases anti-cytokine cytokines, including IL-10 [12].

\section{Conclusion}

In conclusion, several immunomodulatory effects of $S$. striata Boiss on the immune system and possibly management of COVID-19 were discussed; however, the exact effects of all bioactive compounds of S. striata Boiss on cytokine storm and thrombotic issues that frequently occur in critically ill COVID-19 patients require further elucidation. Considering the abundance of quercetin in the extract of the $S$. striata Boiss, this combination and other biological compounds of this plant may have modulating effects on the immune system. It is recommended to conduct clinical trial studies in patients with COVID-19 and other viral diseases.

\section{Ethical Considerations}

\section{Compliance with ethical guidelines}

There were no ethical considerations to be considered in this research.

\section{Funding}

This research did not receive any grant from funding agencies in the public, commercial, or non-profit sectors.

\section{Authors' contributions}

Conceptualization and supervision: Sajjad Jafari and Farhad Seif; Methodology: Sajjad Jafari and Majid Dadmehr; Investigation, writing - original draft, and writing - review \& editing: Sajjad Jafari, Yaeghob Sharifi, Reyhane Rasizadeh; Data collection: Shirin Manshouri, Monireh Kamali, Zahra Vahidi Emami, Data analysis: Sajjad Jafari.

\section{Conflicts of interest}

The authors declared no conflict of interest.

\section{References}

[1] Bahrami M, Kamalinejad M, Latifi SA, Seif F, Dadmehr M. Cytokine storm in COVID-19 and parthenolide: Preclinical evidence. Phytotherapy Research. 2020; 34(10):2429-30. [DOI:10.1002/ptr.6776] [PMID] [PMCID]

[2] Seif F, Aazami H, Khoshmirsafa M, Kamali M, Mohsenzadegan M, Pornour M, et al. JAK inhibition as a new treatment strategy for patients with COVID-19. International Archives of Allergy and Immunology. 2020; 181(6):467-75. [DOI:10.1159/000508247] [PMID] [PMCID]

[3] Roshanravan N, Seif F, Ostadrahimi A, Pouraghaei M, Ghaffari S. Targeting cytokine storm to manage patients with COVID-19: A mini-review. Archives of Medical Research. 2020; 51(7):608-12. [DOI:10.1016/j.arcmed.2020.06.012] [PMID] [PMCID]

[4] Conti P, Caraffa A, Gallenga C, Ross R, Kritas S, Frydas I, et al. Coronavirus-19 (SARS-CoV-2) induces acute severe lung inflammation via IL-1 causing cytokine storm in COVID-19: A promising inhibitory strategy. Journal of Biological Regulators and Homeostatic Agents. 2020; 34(6):1971-5. [DOI:10.23812/20-1-E] [PMID]

[5] Tamri P. A mini-review on phytochemistry and pharmacological activities of Scrophularia striata. Journal of Herbmed Pharmacology. 2019; 8(2):85-9. [DOI:10.15171/jhp.2019.14]

[6] Azadmehr A, Maliji G, Hajiaghaee R, Shahnazi M, Afaghi A. Inhibition of pro-inflammatory cytokines by ethyl acetate extract of Scrophularia striata. Tropical Journal of Pharmaceutical Research. 2012; 11(6):893-7. [DOI:10.4314/tjpr.v11i6.4]

[7] Azadmehr A, Afshari A, Baradaran B, Hajiaghaee R, Rezazadeh S, Monsef-Esfahani H. Suppression of nitric oxide production in activated murine peritoneal macrophages in vitro and ex vivo by Scrophularia striata ethanolic extract. Journal of Ethnopharmacology. 2009; 124(1):166-9. [DOI:10.1016/j. jep.2009.03.042] [PMID] 
[8] Azadmehr A, Hajiaghaee R, Zohal MA, Maliji G. Protective effects of Scrophularia striata in Ovalbumin-induced mice asthma model. Daru: Journal of Faculty of Pharmacy, Tehran University of Medical Sciences. 2013; 21(1):1-7. [DOI:10.1186/2008-2231-21-56] [PMID] [PMCID]

[9] Monsef-Esfahani HR, Hajiaghaee R, Shahverdi AR, Khorramizadeh MR, Amini M. Flavonoids, cinnamic acid and phenyl propanoid from aerial parts of Scrophularia striata. Pharmaceutical Biology. 2010; 48(3):333-6. [DOI:10.3109/13880200903133829] [PMID]

[10] Saeedi-Boroujeni A, Mahmoudian-Sani MR. Anti-inflammatory potential of Quercetin in COVID-19 treatment. Journal of Inflammation. 2021; 18(1):3. [DOI:10.1186/s12950-02100268-6] [PMID] [PMCID]

[11] Endale M, Park SC, Kim S, Kim SH, Yang Y, Cho JY, et al. Quercetin disrupts tyrosine-phosphorylated phosphatidylinositol 3-kinase and myeloid differentiation factor-88 association, and inhibits MAPK/AP-1 and IKK/NF-kBinduced inflammatory mediators production in RAW 264.7 cells. Immunobiology. 2013; 218(12):1452-67. [DOI:10.1016/j. imbio.2013.04.019] [PMID]

[12] Milenković M, Arsenović-Ranin N, Stojić-Vukanić Z, Bufan B, Vučićević D, Jančić I. Quercetin ameliorates experimental autoimmune myocarditis in rats. Journal of Pharmacy and Pharmaceutical Sciences. 2010; 13(3):311-9. [DOI:10.18433/ J3VS3S] [PMID] 